\title{
A New Mechanism for Gravitational Wave Emission in Core-Collapse Supernovae
}

\author{
Christian D. Ott ${ }^{(1)}$, Adam Burrows ${ }^{(2)}$, Luc Dessart ${ }^{(2)}$, Eli Livne ${ }^{(3)}$ \\ (1) Max-Planck-Institut für Gravitationsphysik, Albert-Einstein-Institut, Potsdam, Germany; cott@aei.mpg.de \\ ${ }^{(2)}$ Department of Astronomy and Steward Observatory, The University of Arizona, \\ Tucson,AZ 85721; burrows@zenith.as.arizona.edu,luc@as.arizona.edu and \\ (3) Racah Institute of Physics, The Hebrew University, Jerusalem, Israel; eli@frodo.fiz.huji.ac.il
}

\begin{abstract}
We present a new theory for the gravitational wave signatures of core-collapse supernovae. Previous studies identified axisymmetric rotating core collapse, core bounce, postbounce convection, and anisotropic neutrino emission as the primary processes and phases for the radiation of gravitational waves. Our results, which are based on axisymmetric, Newtonian radiation-hydrodynamics supernova simulations (Burrows et al. 2006), indicate that the dominant emission process of gravitational waves in core-collapse supernovae may be the oscillations of the protoneutron star core. The oscillations are predominantly of g-mode character, are excited hundreds of milliseconds after bounce, and typically last for several hundred milliseconds. Our results suggest that even nonrotating corecollapse supernovae should be visible to current LIGO-class detectors throughout the Galaxy, and depending on progenitor structure, possibly out to Megaparsec distances.
\end{abstract}

PACS numbers: 04.30.Db 04.40.Dg 97.60.Bw 97.60.Jd 97.60.-s

Ever since physicists began to think about gravitational wave detection, core-collapse supernovae have been regarded as prime sources. Traditionally, model calculations estimating the supernova wave signature have focussed on the gravitational-wave emission from rotating iron core collapse and core bounce (see, e.g., [1]). Recent results from stellar evolutionary calculations [2, 3] and neutron star birth spin estimates [4] indicate that presupernova stellar iron cores may rotate much more slowly than previously assumed and that the asphericity during collapse and bounce due to rotation is not generally great enough to produce a sizable time-varying, wave-emitting mass-quadrupole moment. In fact, gravitational radiation from large-scale postbounce convection and anisotropic neutrino emission are likely to exceed the bounce signal of such slowly rotating supernova cores $[1,5,6,6]$.

The first-generation LIGO-class detectors are now operating at design sensitivity and an international network of observatories, including LIGO, GEO600, VIRGO and TAMA, is on-line. Gravitational waves detected from a supernova can provide us with "live" dynamical information from the supernova core, complementing the supernova neutrino pulse as the only other immediate carriers of information from deep inside the star. Using signalprocessing techniques operating on a large set of theoretical templates, it will be possible to extract supernova physics from a sufficiently strong signal [8].

In recent simulations, Burrows et al. 9] have observed that protoneutron star (PNS) core g-modes are excited by turbulence and by accretion downstreams through the unstable and highly-deformed stalled supernova shock (undergoing the Standing-Accretion-Shock Instability [SASI] 9, 10, 11, 12]) at postbounce times of many hundreds of milliseconds. The oscillations damp by the emission of strong sound waves and do not ebb un- til accretion subsides. In this way the core g-modes act as transducers for the conversion of accretion gravitational energy into acoustic power that is deposited in the supernova mantle and, as proposed by Burrows et al. [9], may be sufficient to drive an explosion. Most easily excited is the fundamental $\ell=1$ core $g$-mode, but higher-order eigenmodes and, through nonlinear effects, harmonics of eigenmodes with complicated spatial structures, emerge at later times.

In this letter, we consider the intruiging possibility of the emission of strong gravitational waves from the quadrupole spatial components of the PNS core oscillations. We obtain new estimates for the gravitationalwave signature of core-collapse supernovae. With three different presupernova stellar models, we have performed the longest to date 2D Newtonian radiationhydrodynamics supernova simulations. We find that the gravitational waves from the quadrupole components of the core oscillations dominate the total wave signature in duration, maximum strain, and total energy emission by one to several orders of magnitude. We have also discovered an approximate progenitor dependence: more massive iron cores may experience higher frequency, higher amplitude oscillations, and, hence, more energetic gravitational-wave emission.

Method and Initial Models. We carry out our axisymmetric calculations with the VULCAN/2D code in the multi-group, flux-limited diffusion approximation [9, 13, 14, 15]. The computational grid is comprised of 120 angular zones on a full hemisphere and 160 logarithmicallyspaced, centrally-refined radial zones from $30 \mathrm{~km}$ out to $5000 \mathrm{~km}$. The innermost $30 \mathrm{~km}$ is covered by a Cartesian region that is deformed to smoothly match the polar grid at the transition radius [1]. A resolution test with $50 \%$ more angular and radial zones did not reveal major differences in global integral observables such as the 


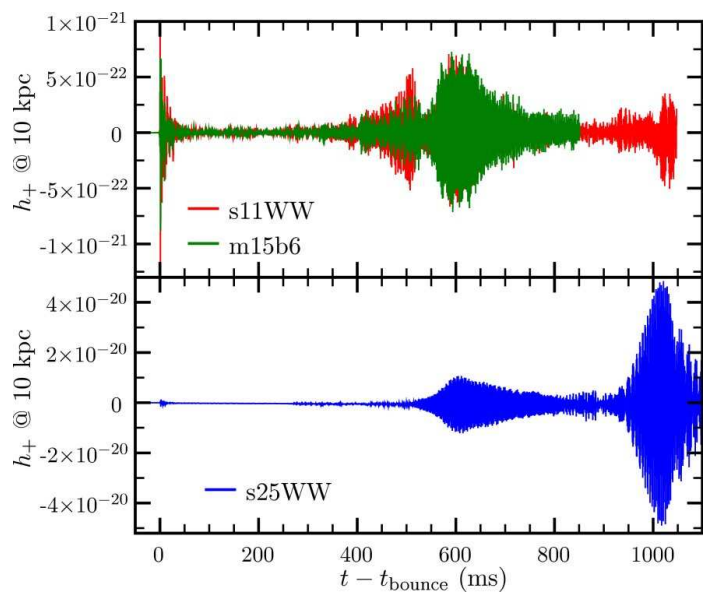

FIG. 1: Dimensionless gravitational-wave strain $\mathrm{h}_{+}$along the equator at a distance of $10 \mathrm{kpc}$. Note that the range of $h_{+}$in the lower panel is almost 50 times wider than that of the top panel.

gravitational-wave strain. We employ the Shen equation of state 16.

We explore three models in this study. Model s11WW is the $11-\mathrm{M}_{\odot}$ (Zero-Age Main Sequence [ZAMS]) presupernova model of Woosley \& Weaver [17] without rotation. Model s25WW is nonrotating as well, but is the $25-\mathrm{M}_{\odot}$ progenitor from the same study. Model m15b6 corresponds to the $15-\mathrm{M}_{\odot}$ progenitor model of Heger et al. 2] which was evolved with a $1 \mathrm{D}$ prescription for rotation and magnetic-field-driven angular momentum redistribution. We map this model onto our $2 \mathrm{D}$ grid under the assumption of constant rotation on cylinders. It has a precollapse ratio of rotational kinetic energy to gravitational potential energy, $\beta=\mathrm{T} /|\mathrm{W}|$, of $\sim 1 \times 10^{-3 \%}$. This value is one to two orders of magnitude smaller than in previous models (e.g., 1, 7, 18]), but yields a PNS consistent with neutron star birth spin estimates [4].

We extract gravitational waves from the mass motions via the quadrupole formula as described in [1, 19, 20]. In addition, we estimate the gravitational-wave emission by anisotropic neutrino radiation with the formalism introduced by [21] and concretized in [6, 20].

Results. Figure 1 depicts the quadrupole gravitational wave strain $h_{+}$as emitted by mass motions scaled to a source distance of 10 kiloparsecs $(\mathrm{kpc})$. In the top panel, we superpose the waveforms of models s11WW and m15b6. Despite the presence of some rotation in the latter and its greater ZAMS mass, the two models have very similar precollapse stellar structures [2, 4, 17]. This is reflected in the very similar shapes of their waveforms. Even though s11WW is not rotating, a bounce burst strain of $\sim 1.3 \times 10^{-21}$ (@ $10 \mathrm{kpc}$ ) is present in our numerical model. The first one to two milliseconds of this burst are the imprint of the transition in grid geometry from the outer polar to the inner Cartesian grids

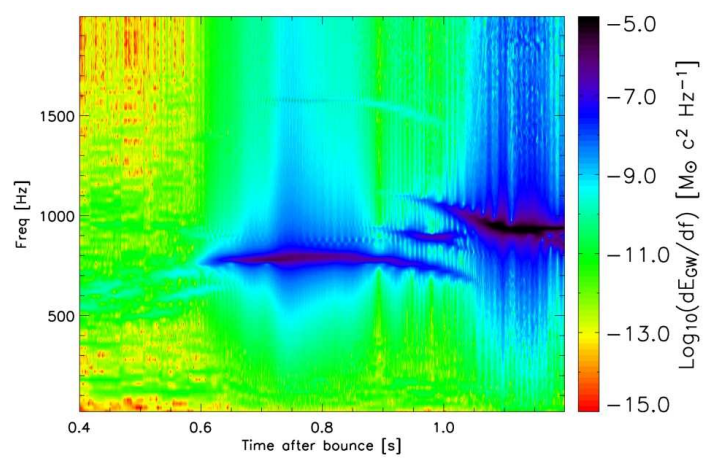

FIG. 2: Frequency-time evolution of model s25WW's gravitational-wave energy spectrum $\left(d E_{\mathrm{GW}} / d f,[20]\right)$ computed with a $50 \mathrm{~ms}$ sampling interval.

which generates a time-varying quadrupole moment at core bounce. It also induces initial perturbations for vortical motion in the Ledoux-unstable regions behind the expanding shock that sets in almost immediately and with a perhaps too fast initial growth rate after core bounce. The amount of rotational energy in m15b6's core (at bounce, $\beta \lesssim 0.02 \%$ and at the end, $\beta \sim 0.08 \%$ ) is too small to have a large influence on the core dynamics and, thus, on the waveform, except to slightly stabilize the aspherical fluid motion at and shortly after bounce. In both models, until about $\sim 250 \mathrm{~ms}$ after bounce the physical waveform is dominated by convective motions in the PNS and in the post-shock region. As the SASI 9, 10, 11, 12]) becomes vigorous and leads to global deformation of the standing shock (see, e.g., [9]) the wave emission from the post-shock flow increases.

As described in Burrows et al. [9] the fundamental core g-mode $(\ell=1, f \sim 330 \mathrm{~Hz}$ in s11WW and $\mathrm{m} 15 \mathrm{~b} 6)$ is excited by turbulence and accretion. It grows strong around $\sim 400 \mathrm{~ms}$ after bounce and starts transferring energy to the harmonic at $2 f$ through nonlinear effects. This is reflected in the rise of s11WW's gravitational-wave strain around that time. $h_{+}$reaches a local maximum, then quickly decays to about one-third that amplitude, only to pick up again after some tens of milliseconds, rising to even higher amplitudes (a maximum of $\sim 7 \times 10^{-22}$ [@ $10 \mathrm{kpc}]$ ), followed by a quasi-exponential decay with a $\sim 100 \mathrm{~ms} e$-folding time. We attribute the gravitationalwave emission in these two 'humps' to the quadrupole spatial component of the $2 f$ harmonic of the $\ell=1$ core gmode. s11WW's gravitational-wave energy spectrum exhibits prominent emission in a band around $\sim 650 \mathrm{~Hz}$. A frequency analysis shows that the harmonic appears first at a frequency of $\sim 590 \mathrm{~Hz}$, which increases over $200 \mathrm{~ms}$ to a maximum of about $680 \mathrm{~Hz}$, and then continuously decreases to $\sim 500 \mathrm{~Hz}$ at the end of the simulation. In this way, the gravitational-wave emitting component exactly mirrors the behavior of the $\ell=1$ g-mode which goes through the same phases [9, 22]. This behavior is qual- 
itatively consistent with the g-mode frequency evolution in PNSs presented in 23], who used general relativity and obtained more compact PNSs with higher-frequency modes. Interestingly, we find that the time of the first lull in the wave emission ( $\sim 500 \mathrm{~ms})$ coincides with the point in time at which the core-oscillation mode reaches its maximum frequency and its frequency derivative is zero [22]. Even though model s11WW begins to explode around $550 \mathrm{~ms}$, its core oscillation does not subside immediately [9].

\begin{tabular}{|c|c|c|c|c|c|}
\hline Model & $\begin{array}{l}\Delta \mathrm{t}^{a} \\
(\mathrm{~ms})\end{array}$ & $\begin{array}{c}\left.\mathrm{h}_{+, \text {max }}\right|^{b} \\
\left(10^{-21}\right)\end{array}$ & $\begin{array}{c}\mathrm{h}_{\text {char,max }}^{b, c} \\
\left(10^{-21}\right)\end{array}$ & $\begin{array}{c}\mathrm{f}\left(\mathrm{h}_{\mathrm{char}, \max }\right) \\
(\mathrm{Hz})\end{array}$ & $\begin{array}{c}\mathrm{E}_{\mathrm{GW}}^{d} \\
\left(10^{-7} \mathrm{M}_{\odot} \mathrm{c}^{2}\right)\end{array}$ \\
\hline s11WW & 1045 & 1.3 & 22.8 & 654 & 0.16 \\
\hline s25WW & 1110 & 50.0 & 2514.3 & 937 & 824.28 \\
\hline m15b6 & 927.2 & 1.2 & 19.3 & 660 & 0.14 \\
\hline
\end{tabular}

${ }^{a}$ time between bounce and the end of the simulation

${ }^{b}$ at $10 \mathrm{kpc} \quad{ }^{c}$ see Eq. (5.3) of [24. ${ }^{d}$ see Eq. (12) of 20]

The lower panel of Fig. 1 displays model s25WW's waveform. s25WW's precollapse stellar structure is significantly different from those of s11WW and m15b6. Most importantly, its iron core is more massive $\left(1.92 \mathrm{M}_{\odot}\right.$ vs. $1.37 \mathrm{M}_{\odot}$ and $1.47 \mathrm{M}_{\odot}$, respectively) and more extended. These differences in progenitor structure lead to different postbounce evolution for model s25WW. Its initial shock radius is significantly smaller and the SASI becomes vigorous some $100 \mathrm{~ms}$ later than in the two other models 25]. Figure 2] shows a frequency-time plot of s25WW's gravitational-wave energy spectrum starting at $400 \mathrm{~ms}$ after bounce. The first burst of gravitational waves, starting at about $500 \mathrm{~ms}$ and slowly fading afterwards (Fig. 1), is centered about $\sim 800 \mathrm{~Hz}$. In this model, the $\ell=1$ fluid mode, which dominates the dynamics at that time, is centered at $\sim 400 \mathrm{~Hz}$ and as for models s11WW and m15b6, we identify the wave-emitting component as part of the harmonic at $2 f$ of the former. At $\sim 900 \mathrm{~ms}$ after bounce, much stronger waves begin to be emitted through the excitation of an $\ell=2$ core eigenmode (Fig. 22 at that time $f \sim 950 \mathrm{~Hz}$ ). It reaches a maximum strain of $\sim 5 \times 10^{-20}$ (@ $10 \mathrm{kpc}$ ) and lasts for at least $200 \mathrm{~ms}$, emitting a total of close to $10^{-4} \mathrm{M}_{\odot} \mathrm{c}^{2}$ $\left(\simeq 1.8 \times 10^{50} \mathrm{erg} !\right)$ in gravitational waves (Fig. [3).

For the three models considered here we find the contributions of anisotropic neutrino emission to be completely negligible compared with those of the core oscillations. Extracting the neutrino-flux anisotropies at an observer radius of $200 \mathrm{~km}$, well outside the neutrinospheres, and extrapolating to $10 \mathrm{kpc}$ distance we find maximum strains of $5.5 \times 10^{-23}, 2.6 \times 10^{-23}$, and $1.3 \times 10^{-23}$ for $\mathrm{s} 25 \mathrm{WW}$, s11WW, and m15b6, respectively. These numbers are also up to five times smaller than previous estimates obtained with codes that perform neutrino transport along rays, which tends to overemphasize anisotropies [6, 7, 20], while our flux-limited diffusion approach tends to smooth them out slightly. Due to their

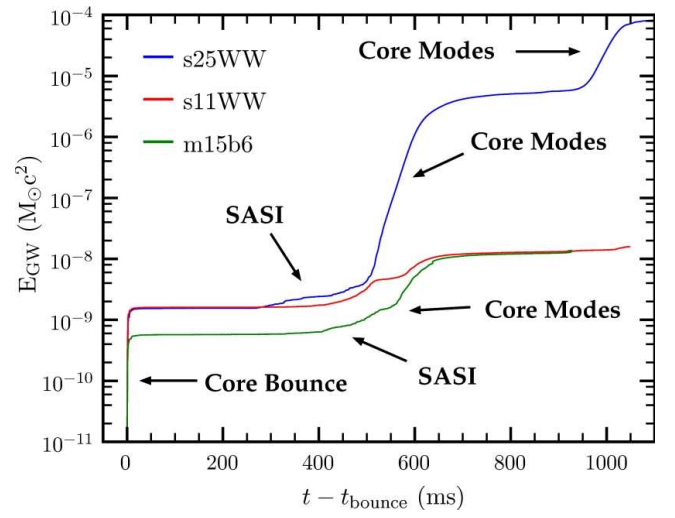

FIG. 3: Integrated energy emitted in gravitational waves.

low-frequency character, gravitational waves from neutrinos may not be observable by Earth-based detectors

Summary. We have derived and analyzed the gravitational-wave emissions in long-term Newtonian 2D radiation-hydrodynamics supernova calculations for three different progenitors, one of which includes angular momentum consistent with the inferred rotation period of young pulsars. We find that the gravitational-wave emissions of all models are dominated by the quadrupolar components of the core g-mode oscillations first discovered by Burrows et al. [9]. If the core oscillations seen in simulations do obtain, their gravitational-wave emission will exceed the emission from all previously considered emission processes, including rotational core bounce, convection, and anisotropic neutrino emission. Importantly, we point out that the excitation of the core g-modes does not depend on the particular explosion mechanism proposed by Burrows et al. [9], but is likely to be a generic phenomenon induced by turbulence and accretion downstreams in any suitably delayed explosion mechanism.

The recent study of Müller et al. 7] suggests a total energy emission of $3 \times 10^{-9} \mathrm{M}_{\odot} \mathrm{c}^{2}$ for a rotating model (initially about 3 times as fast as our m15b6). For our nonrotating, low-mass model s11WW we find about 5 times as much energy emission: $1.6 \times 10^{-8} \mathrm{M}_{\odot} \mathrm{c}^{2}$. For the slowly rotating, but otherwise very similar model m15b6, we calculate a total gravitational-wave energy of $1.4 \times 10^{-8} \mathrm{M}_{\odot} \mathrm{c}^{2}$ (Fig. 3 and Table 1). For the initial rotation rate and angular momentum distribution present in m15b6, we do not find significant qualitative or quantitative differences caused by such slow rotation. Model s25WW, due to its more massive iron core, higher postbounce accretion rates, and higher pulsation frequencies and amplitudes, emits an amazing $8.2 \times 10^{-5} \mathrm{M}_{\odot} \mathrm{c}^{2}$, almost one tenth of a typical supernova explosion energy. Clearly, radiation-reaction effects, not included in our present work, might be sig-

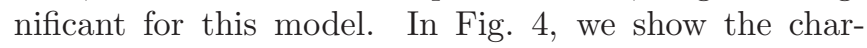
acteristic gravitational-wave strain spectra of the three models, if located at $10 \mathrm{kpc}$. $h_{\mathrm{char}}$ is defined by [24] as $h_{\text {char }}(f)=D^{-1} \sqrt{2 \pi^{-2} G c^{-3} d E_{\mathrm{GW}} / d f}$, where $D$ is the dis- 


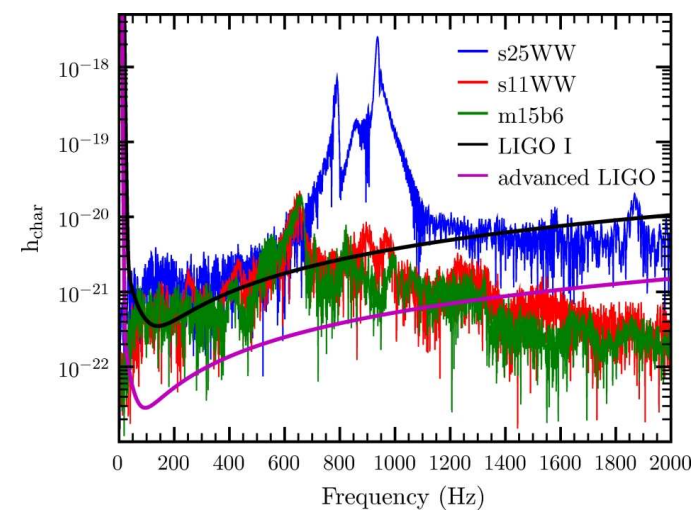

FIG. 4: Characteristic strain spectra contrasted with initial and advanced LIGO (optimal) rms noise curves.

tance to the source. This is a particularly useful measure, since it incorporates the amount of energy radiated in a spectral interval $d f$ around $f$. In addition, we show the optimal rms noise strain $\left(h_{\mathrm{rms}}=\sqrt{f S(f)}, S(f)\right.$ being the spectral strain sensitivity) of both LIGO I and Advanced LIGO [26]. All $\mathrm{h}_{\text {char }}$ spectra peak strongly at the frequencies identified with the quadrupole components of the core oscillations (Fig. 2] between 600 and $1000 \mathrm{~Hz}$, likely to be higher when general relativity is included), corroborating the narrow-band nature of the emission process. Given our results, we conclude that, if the core oscillations observed in our simulations are generically excited in core-collapse supernovae, even nonrotating supernovae of small to intermediate progenitor mass should be observable by LIGO throughout the Milky Way and beyond. Massive progenitors could be detectable out to $\sim 100$ times greater distances and their prolonged and extremely energetic core-oscillation wave signature might be the generic precursor of stellar-mass black-hole formation.

We point out that the work presented here is based on simulations in 2D Newtonian gravity and only quadrupole wave emission has been considered. General relativity is likely to increase the frequency of the PNS eigenmodes, but is unlikely to lead to qualitative differences. Fast rotation might lead to the partial stabilization of the post-shock convection, might affect the growth of core oscillations, and will likely lead to nonaxisymmetric rotational instabilities for $\beta \gtrsim 8 \%$ [27]. In $3 \mathrm{D}$, the temporal and spatial mode and SASI structures may change.

We acknowledge help from and discussions with C. Meakin, J. Murphy, H. Dimmelmeier, E. Müller, J. Pons, N. Stergioulas, L. Rezzolla, and E. Seidel. We acknowledge support from the Scientific Discovery through Advanced Computing (SciDAC) program of the DOE, grant number DE-FC02-01ER41184, and from the NSF, grant number AST-0504947. E. L. acknowledges support from the Israel Science Foundation under grant 805/04. This research used resources of the National Energy Research Scientific Computing Center, which is supported by the Office of Science of the U.S. Department of Energy under Contract No. DE-AC03-76SF00098.

[1] C. D. Ott, A. Burrows, E. Livne, and A. Walder, Astrophys. J. 600, 834 (2004).

[2] A. Heger, S. E. Woosley, and H. C. Spruit, Astrophys. J. 626, 350 (2005).

[3] R. Hirschi, G. Meynet, and A. Maeder, Astron. Astrophys. 425, 649 (2004).

[4] C. D. Ott, A. Burrows, T. A. Thompson, E. Livne, and R. Walder, Astrophys. J. Suppl. Ser., 164, 130 (2006).

[5] H.-T. Janka and E. Müller, Astron. Astrophys. 306, 167 (1996).

[6] A. Burrows and J. Hayes, Phys. Rev. Lett. 76, 352 (1996).

[7] E. Müller, M. Rampp, R. Buras, H.-T. Janka, and D. H. Shoemaker, Astrophys. J. 603, 221 (2004).

[8] T. Summerscales, A. Burrows, L. S. Finn, and C. D. Ott, in preparation (2006).

[9] A. Burrows, E. Livne, L. Dessart, C. D. Ott, and J. Murphy, Astrophys. J., 640, 878, (2006).

[10] T. Foglizzo, Astron. Astrophys. 368, 311 (2001).

[11] J. M. Blondin, A. Mezzacappa, and C. DeMarino, Astrophys. J. 584, 971 (2003).

[12] R. Buras, H.-T. Janka, M. Rampp, and K. Kifonidis, Astron. Astrophys., submitted, astro-ph/0512189 (2005).

[13] E. Livne, A. Burrows, R. Walder, I. Lichtenstadt, and T. A. Thompson, Astrophys. J. 609, 277 (2004).

[14] R. Walder, A. Burrows, C. D. Ott, E. Livne, I. Lichtenstadt, and M. Jarrah, Astrophys. J. 626, 317 (2005).

[15] L. Dessart, A. Burrows, E. Livne, and C. D. Ott, Astrophys. J., accepted, astro-ph/0510229 (2006).

[16] H. Shen, H. Toki, K. Oyamatsu, and K. Sumiyoshi, Progress of Theoretical Physics 100, 1013 (1998).

[17] S. E. Woosley and T. A. Weaver, Astrophys. J. Suppl. Ser. 101, 181 (1995).

[18] H. Dimmelmeier, J. Font, and E. Müller, Astron. Astrophys. 393, 523 (2002).

[19] L. S. Finn and C. R. Evans, Astrophys. J. 351, 588 (1990).

[20] E. Müller and H.-T. Janka, Astron. Astrophys. 317, 140 (1997).

[21] R. Epstein, Astrophys. J. 223, 1037 (1978).

[22] A. Burrows, E. Livne, L. Dessart, C. D. Ott, and J. Murphy, in proceedings of, "Astronomy with Radioactivities V: From Gamma-Rays to Stardust," held at Clemson University, SC, USA (2005).

[23] V. Ferrari, G. Miniutti, and J. A. Pons, Mon. Not. R. Astron. Soc. 342, 629 (2003).

[24] É. É. Flanagan and S. A. Hughes, Phys. Rev. D 57, 4535 (1998).

[25] A. Burrows, E. Livne, L. Dessart, C. D. Ott, and J. Murphy, in preparation (2006).

[26] E. Gustafson, D. Shoemaker, K. Strain, and R. Weiss, LSC white paper on detector research and development, Technical Report, LIGO T99080-00-D (1999).

[27] C. D. Ott, S. Ou, J. E. Tohline, and A. Burrows, Astrophys. J. Lett. 625, L119 (2005). 